\title{
Generic Concept to Program the Time Domain of Self-Assemblies with a Self-Regulation Mechanism
}

\author{
Thomas Heuser, Ann-Kathrin Steppert, Catalina Molano Lopez, Baolei Zhu, and Andreas Walther* \\ DWI-Leibniz-Institute for Interactive Materials, Forckenbeckstraße 50, 52074 Aachen, Germany
}

Supporting Information
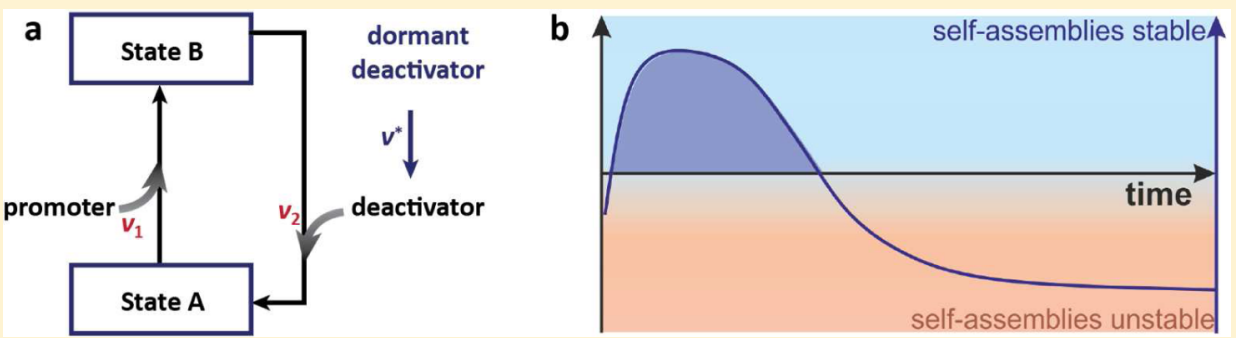

ABSTRACT: Nature regulates complex structures in space and time via feedback loops, kinetically controlled transformations, and under energy dissipation to allow non-equilibrium processes. Although man-made static self-assemblies realize excellent control over hierarchical structures via molecular programming, managing their temporal destiny by self-regulation is a largely unsolved challenge. Herein, we introduce a generic concept to control the time domain by programming the lifetimes of switchable self-assemblies in closed systems. We conceive dormant deactivators that, in combination with fast promoters, enable a unique kinetic balance to establish an autonomously self-regulating, transient $\mathrm{pH}$-state, whose duration can be programmed over orders of magnitude-from minutes to days. Coupling this non-equilibrium state to $\mathrm{pH}$-switchable self-assemblies allows predicting their assembly/disassembly fate in time, similar to a precise self-destruction mechanism. We demonstrate a platform approach by programming self-assembly lifetimes of block copolymers, nanoparticles, and peptides, enabling dynamic materials with a self-regulation functionality.

KEYWORDS: Non-Equilibrium Self-Assembly, Self-Regulation, Programmable Materials, Time Domain, Switchable Materials, Dynamic Properties

$\mathrm{T}$ he recent decade has witnessed great progress in the static near-equilibrium self-assembly, and we increasingly understand how to construct very complex soft matter via molecular programming using competing interactions, coassemblies, and hierarchical length scales. ${ }^{1-17}$ This has furnished real-life materials with unprecedented functionalities, inaccessible without balanced interplay of molecular interactions and delicate control over nano/mesostructuration. ${ }^{18-22}$ However, reaching new levels of functionalities and complexity requires to go beyond structuring under static conditions and to find pathways to orchestrate the time domain of self-assemblies via internal and external feedback mechanisms. Success in this largely unsolved challenge would open up possibilities for selfregulating, dynamic, and adaptive behavior.

The challenge to control the time domain of self-assemblies can be approached by managing the kinetics of opposing assembly/disassembly reactions, the implementation of internal feedback systems or the use of energy dissipation to sustain structures only as long as a chemical fuel is available. The most notable approach toward temporal patterns uses the coupling of specifically designed self-assemblies to nonlinear chemical oscillators. Most success was reported using the BelousovZhabotinsky reaction to generate pulsating hydrogels or control aggregate formation. ${ }^{23}$ Such nonlinear oscillators are, however, very rare and attempts to tune their time scale quickly lead to collapse of their feedback systems. ${ }^{23-28}$

Dissipative self-assemblies are forcefully kept away from equilibrium by continuous energy input. ${ }^{29-32}$ They are prominent in nature (e.g., microtubules) but profoundly difficult to create artificially. ${ }^{30-32}$ Most difficult to realize are chemically fuelled dissipative self-assemblies in closed systems. These require establishing a delicate balance between a promoting $\left(v_{1}\right)$ and a competing deactivating $\left(v_{2}\right)$ step to allow a nonlinear evolution with the formation of a transient self-assembled state that self-regulates/decays without any further external signal (Scheme 1). In some cases, the deactivating step may just be a slow hydrolysis of an activated species. They are particularly rare as tedious optimization is needed to prevent direct annihilation of the promoter in the deactivating environment, and the boundary condition of $v_{1}>$ $v_{2}$ for transient self-assembly formation remains an outstanding challenge (Figure 1). ${ }^{32,33}$ For instance, van Esch and coworkers used MeI to temporally methylate carboxyl acid functions of a gelator and trigger a self-assembly that is subsequently reversed by hydroxide-triggered hydrolysis. ${ }^{32}$

Received: July 10, 2014

Published: November 13, 2014 
Scheme 1. Kinetic control to program self-regulating self-assemblies in time by simultaneous injection of rapid promoters and dormant deactivators (DDs). (a) Stringent kinetic boundary conditions $v_{1} \gg v_{2}$ to achieve transient self-assemblies can be lifted using DDs. The relevant kinetic condition changes to $v_{1} \gg v^{*}, v_{1} \approx v_{2}$ becomes irrelevant. Ultimately, an excess of the deactivator leads to the self-regulated disassembly. (b) Simultaneous addition of promoter and DD leads to a transient pH profile (red), which the self-assembly follows with system-specific offsets $\left(t_{\mathrm{d} 1}, t_{\mathrm{d} 2}\right)$
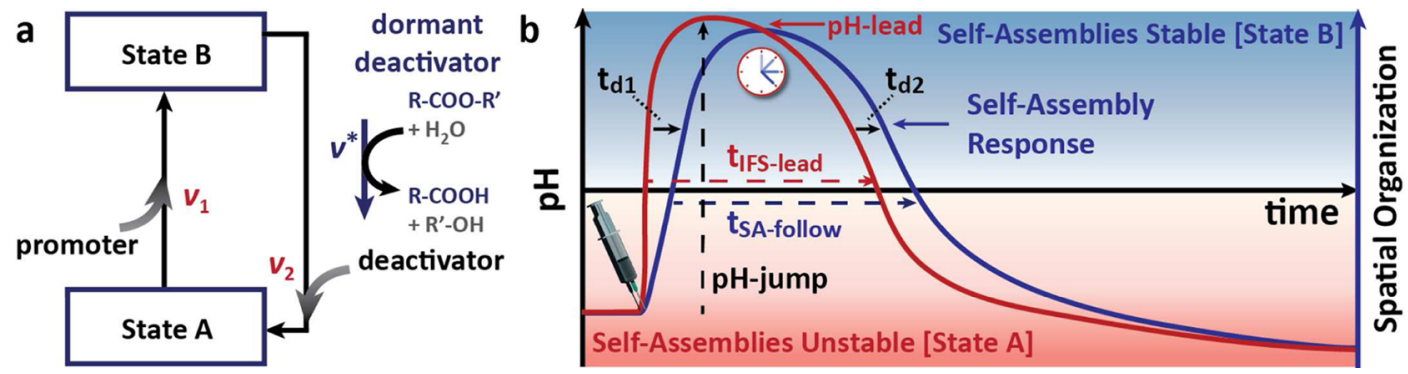

a

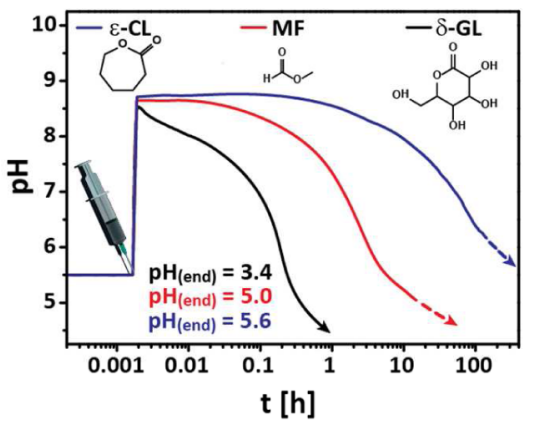

b

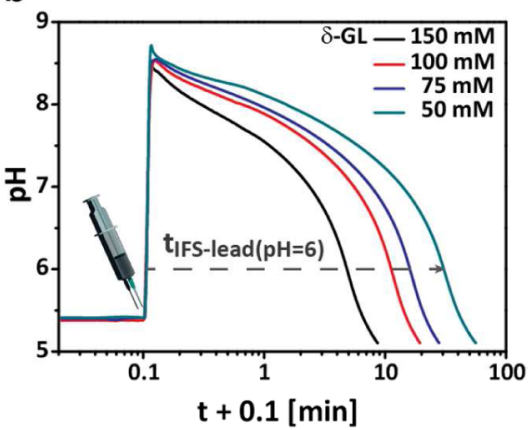

C

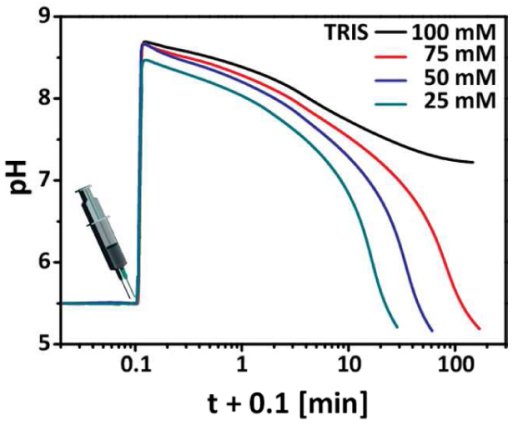

Figure 1. Programming the time scales of transient $\mathrm{pH}$ profiles. (a) Molecular structure of the DD controls the hydrolysis kinetics (ring strain and electronic activation) and regulates the time scale from minutes $(\delta$-GL) to hours $(\mathrm{MF})$ to days $(\varepsilon-\mathrm{CL})$. Conditions: $20 \mathrm{mM}$ TRIS (pH $=8.8)$ as promoter and $100 \mathrm{mM}$ DD. (b) DD concentration controls the decay kinetics at constant promoter concentration. Conditions: $20 \mathrm{mM}$ TRIS (pH = 8.8) as promoter and different concentrations of $\delta$-GL as DD. (c) Buffer capacity regulates the plateau length of the transient $\mathrm{pH}$ profile at constant DD concentration. Conditions: Different concentrations of TRIS $(\mathrm{pH}=8.8)$ as promoter and $100 \mathrm{mM} \delta$-GL as DD.

Overall, the need for system-specific optimization is the major bottleneck to push sophisticated chemically fuelled dissipative self-assemblies into applications.

Such challenging, non-equilibrium concepts with potential internal control of the temporal fate contrast classical stimuli responsiveness in static near-equilibrium self-assembly, which is the conceptual cornerstone of present-day switchable advanced materials based on external triggers (details in Figure SI1, Supporting Information). For a simple responsive system, the "on" switch (promoter) induces a change from state A to state B. State B remains infinitely stable in a closed system. Hence, self-regulation is not found and the time domain does not feature autonomous control. Only once the "off" switch is triggered externally (deactivator), the state B returns to state A. If both switches are added simultaneously (e.g., acid plus base; heating plus cooling), there is no change in the system as the two triggers basically annihilate instantaneously $\left(v_{1}=v_{2}\right)$.

However, appreciating the differences between sophisticated dissipative self-assemblies, self-oscillating reactions, and classical responsive materials allows to identify emerging possibilities arising at their interface. This may bring forward new types of facile self-assembly concepts and materials with more dynamic properties and internal control over the temporal fate, mainly empowered by mastering kinetic control over activating and deactivating steps.

To this end, we herein introduce a facile and generic approach to achieve temporally programmed, self-regulating, self-assembling systems by playing a kinetic trick and by precisely modulating their surrounding. We take charge of the activating and deactivating pathway and conceive "dormant deactivators" (DD), which slowly decay $\left(v^{*}\right)$ and liberate an active deactivator (Scheme 1a). Dormant is defined ${ }^{34}$ as a physical function, here the deactivation, being suspended or slowed down. This simplifies the kinetic boundary conditions to achieve a transient self-assembling state upon simultaneous addition of both activator and dormant deactivator to $v_{1} \gg v^{*}$. During the preprogrammed course, the self-assemblies form (fast promoter) and then decay (dormant deactivator). We demonstrate that the duration of this transient non-equilibrium state can be tuned over four orders of magnitude, from minutes to days, similar to encoding a precise self-destruction mechanism.

We focus on a transient change of $\mathrm{pH}$ in the alkaline regime using DDs slowly hydrolyzing to acid (Scheme 1b). The simultaneous injection with a fast promoter, that is, a directly available base, creates a kinetically controlled transient $\mathrm{pH}$-state with a fast rise and a controlled decay (due to an ultimate excess of DD). The hydrolysis speed of the $\mathrm{DD}$ is $\mathrm{pH}$ dependent and the combination with a rapid promoter increase the $\mathrm{pH}$ and triggers an accelerated decomposition. This leads to an overall negative feedback. Hence, we term the full system a simple yet efficient internal feedback system (IFS) that can be coupled to virtually all $\mathrm{pH}$-triggered self-assemblies operating in the targeted $\mathrm{pH}$ regime. We use the term internal to clearly express that the system operates in closed conditions and without the need of another external trigger for disassembly as 
a

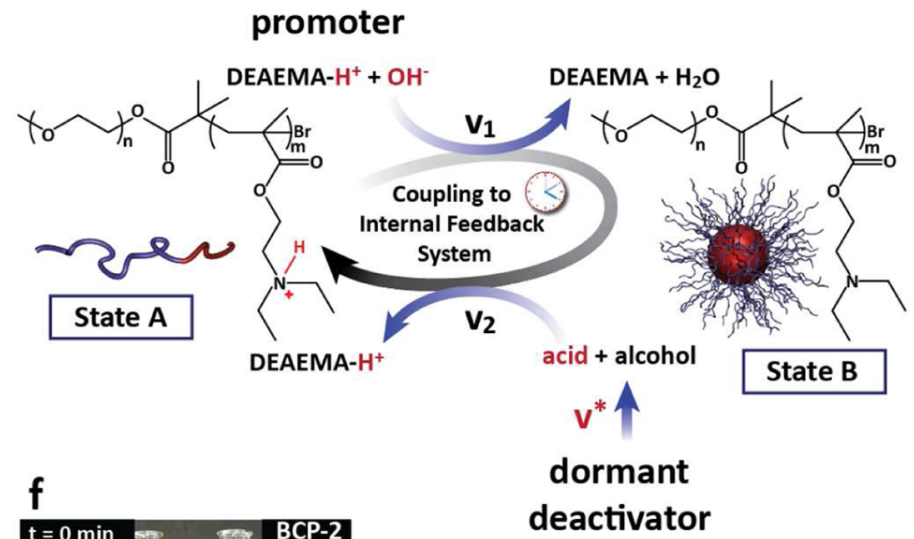

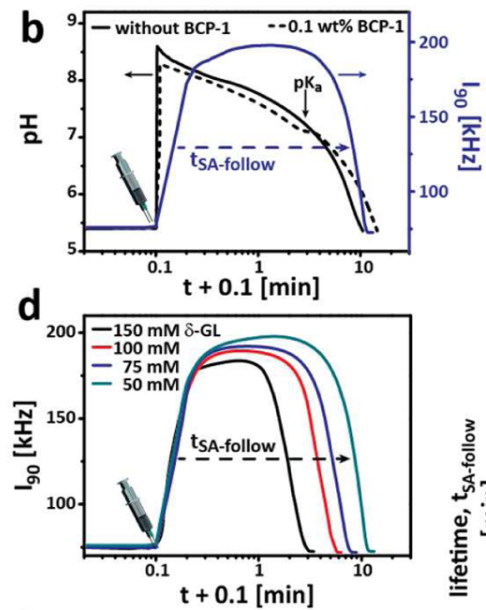

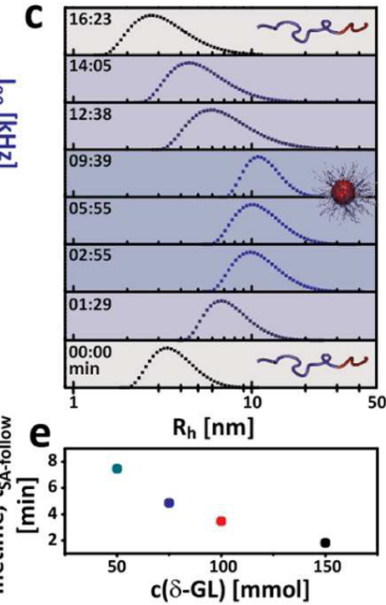

g

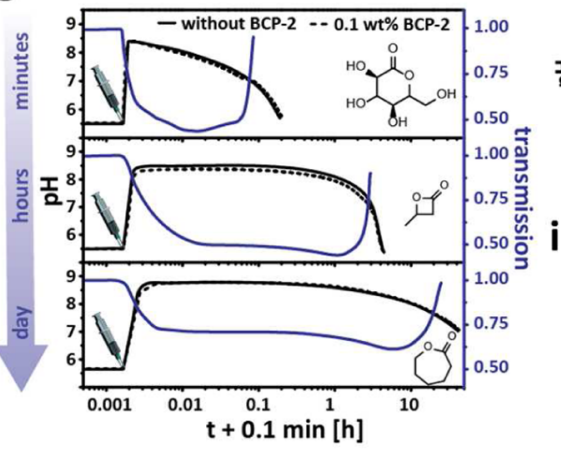

h

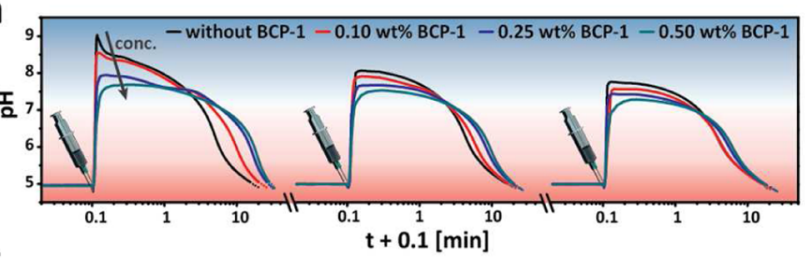

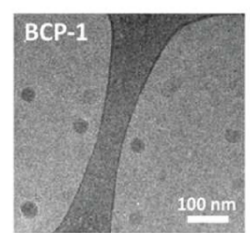
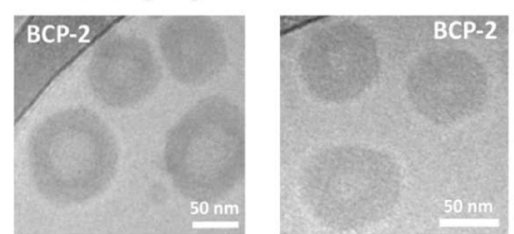

Figure 2. Temporal programming of self-regulating BCP self-assemblies using IFSs. BCP concentration is 0.1 wt \%. (a) Unimer-to-micelle-to-unimer transition upon deprotonation of DEAEMA- $\mathrm{H}^{+}$in BCP-1 due to promoter consumption and subsequent dynamic reprotonation by deactivator consumption. (b) Unimer-to-micelle-to-unimer transition as a response to an IFS for BCP-1, tracked by pH measurement and static LS at $90^{\circ}\left(I_{90^{\circ}}\right)$, and (c) hydrodynamic radii distributions. Conditions: $20 \mathrm{mM}$ TRIS $(\mathrm{pH}=8.8) / 50 \mathrm{mM} \delta$-GL. (d,e) DD concentration regulates the lifetime of the transient micellar state. Conditions: $20 \mathrm{mM}$ TRIS $(\mathrm{pH}=8.8$ )/various concentrations of $\delta$-GL. (f) Time-laps snapshot series of four temporally programmed vesicle self-assemblies of BCP-2. Conditions: $100 \mathrm{mM}$ TRIS ( $\mathrm{pH}=8.8$ ) and 90, 100, 150, 300 mM $\delta$-GL (left to right, Supporting Information Movie SI1). (g) Order-of-magnitude changes of the lifetimes of vesicles controlled by the chemical structure of the DD. Conditions: 100 $\mathrm{mM}$ TRIS ( $\mathrm{pH}=8.8$ )/300 mM of various DDs as indicated. (h) Repeated jumps for different concentrations of BCP-1. Conditions: $20 \mathrm{mM}$ TRIS $(\mathrm{pH}=8.8) / 100 \mathrm{mM} \delta$-GL. (i) Cryo-TEM images of micellar (BCP-1) and vesicular (BCP-2) morphologies.

in classical responsive self-assembly. This enables a unique platform concept to temporally program self-assemblies in closed systems over at least four orders of magnitude in time, from minutes to days. We showcase temporal programming for block copolymer self-assemblies (micelles and vesicles), and extend it as a generic platform to nanoparticles and peptides, all being key building blocks for advanced nanoscale and molecular materials. $2,5,11,18,35$

The key ingredients to our platform approach are DDs; dormant expresses a retarded physical function. ${ }^{34}$ These are selected from ester-containing molecules that slowly hydrolyze and furnish acid ( $v^{*}$, active deactivators; Supporting Information Figure SI2). Their structure controls the hydrolysis rate and acidity ( $\mathrm{p} K_{\mathrm{a}}$ value) and governs the $\mathrm{pH}$-decay kinetics and final $\mathrm{pH}$ together with their concentration (Figure 1). The magnitude of the $\mathrm{pH}$ jump depends on the amount and type of added base, and alkaline buffers can be used as promoter to extend the plateau. Suitable promoters can be selected from a wide range of basic buffers if ion-specific effects need to be considered. Figure 1a depicts the influence of molecular structure for three DDs. Simultaneous injection of $100 \mathrm{mM}$ $\mathrm{DD}$ and $20 \mathrm{mM}$ TRIS buffer $\mathrm{pH}=8.8$ (fast promoter; TRIS = tris(hydroxymethyl)aminomethane hydrochloride) leads to a transient alkaline $\mathrm{pH}$-state with a rapid decay within minutes for gluconic acid $\delta$-lactone $(\delta$-GL), whereas methyl formate (MF) decays within hours, and $\varepsilon$-caprolactone $(\varepsilon-\mathrm{CL})$ requires days.

$\delta$-GL is used in food industry and was applied earlier for shear-free acidification of solutions in a linear gradient. ${ }^{36,37}$ Most notable is the work by Adams ${ }^{37,38}$ targeting a more homogeneous gelation of dipeptides at low $\mathrm{pH}$ by slow acidification using $\delta$-GL. Such a linear decrease is only able to trigger one transition, assembly or disassembly, and results in a rather similar process and properties compared to switching states in classical static self-assemblies. However, the combination with a fast promoter allows a new level of kinetic balance and enables a spike that, when coupled to a self-assembly operating in this transient $\mathrm{pH}$-regime, allows it to pass two phase transitions consecutively and with high confidence (assembly/disassembly), furnishing a transient, intermediate self-assembly with a programmable time signature and encoded lifetimes of the self-assemblies. Self-regulation can be achieved with high fidelity and for various self-assembling systems.

Besides different DDs, a wide tunability is given by varying the ratio of promoter to DD. Longer plateau times occur upon lowering the concentration of the DD at constant buffer/ promoter strength (Figure 1b). Similarly, an increase in the buffer/promoter capacity at constant DD concentration also 
leads to longer plateau times (Figure 1c). Excess buffer prevents a decay. In summary, the duration of this transient state, $t_{\mathrm{IFS} \text {-lead, }}$ depends on the concentration and structure of the DD and can be substantially prolonged and tailored to a desired $\mathrm{pH}$ level using specific buffers as promoter and increasing the ratio of promoter to DD. For successful temporal programming $\left(t_{\mathrm{SA} \text {-follow }}\right)$, the $\mathrm{pH}$-switchable self-assemblies need to be in state $\mathrm{A}$ and couple with a phase transition within the $\mathrm{pH}$ jump to temporally reach state $\mathrm{B}$. Kinetic delays occur $\left(t_{\mathrm{d} 1}\right.$ and $\left.t_{\mathrm{d} 2}\right)$ depending on the particular self-assembling system (Scheme $1 \mathrm{~b})$.

It is important to distinguish our approach from chemical clocks or self-oscillating reactions, both in terms of approach and possible application. Self-oscillating systems only operate in a narrow window and a tuning of the periodicity of the oscillations over orders of magnitude is near impossible to achieve. ${ }^{23,24,26-28,39}$ Additionally, typical continuous $\mathrm{pH}$ oscillators are open and require constant feeding of reactants. $^{24-28,40}$ Even though the chemistry of $\mathrm{pH}$ oscillators would in principal also allow creating a primary transient state upon a single injection, the accessible time scales and $\mathrm{pH}$ regime remain limited. For instance, we did not succeed in establishing reliable time scales for the methylene glycol-sulfite system. $^{40}$ Moreover, these $\mathrm{pH}$ oscillators typically contain oxidants, which may in principal interfere with more delicate self-assembling systems susceptible to oxidation in the range of the oxidation potentials of the oscillator. Therefore, a simultaneous control over the time scale spanning four orders of magnitude and of the $\mathrm{pH}$ jump height (herein crossing seven $\mathrm{pH}$ units; see below) requires going beyond the possibilities of established oscillators and motivates exploring the tunability and capabilities of our benign activator/dormant deactivator system. The major advantage is that simple exchange of the DD allows to change the time scales completely while keeping other parameters constant due to unlike activation for hydrolysis. Second, and more importantly, the potential applications are completely different. While oscillating reactions yield pulsating systems, ${ }^{23}$ useful for hydrodynamic pumping, for example, our targeted programmable state focuses on generating transient self-regulating states and time-programmed memory effects into materials.

Conceptually, our systems are different than chemical dissipative self-assemblies, by having the whole system (aqueous environment plus self-assembling material) out-ofequilibrium, whereas only the self-assembling material is out-ofequilibrium in dissipative self-assemblies.

We first demonstrate temporal programming using our IFS approach for block copolymer solution self-assemblies (BCPs). $\mathrm{BCPs}$ are remarkably interesting materials, combining facile synthesis and switchability with hierarchical self-assembly and applications, for example, in nanolithography, photonics, hydrogels, or drug delivery. $6,8,11,19-21,35$

We synthesized two different $\mathrm{pH}$-switchable BCPs: EO114$b$-DEAEMA99 (BCP-1, $M_{\mathrm{n}}=26000 \mathrm{~g} / \mathrm{mol}$ ) and POEGMA9$b$-DEAEMA224 (BCP-2; $M_{\mathrm{n}}=46000 \mathrm{~g} / \mathrm{mol}$. Numbers denote degree of polymerization; EO, poly(ethylene oxide); OEGMA, poly(oligoethylene oxide methacrylate); DEAEMA, poly(diethylaminoethyl methacrylate)). EO and OEGMA are fully water-soluble at room temperature, whereas DEAEMA turns solvophobic/insoluble at $\mathrm{pH}>7.0$ (Supporting Information Figure SI3). ${ }^{41}$ This allows switchable self-assemblies with a transition intended to fit our IFS. We designed the BCPs according to their DEAEMA weight fractions to be in the star- like (BCP-1) and crew-cut (BCP-2) regime to target spherical micelles and vesicles. ${ }^{42}$

Simultaneous addition of promoter and DD allows programmable autonomous self-regulation of the corresponding BCP self-assemblies in time (Figure 2a). We combine in-situ light scattering with $\mathrm{pH}$ measurements to analyze the nonlinear evolution self-assemblies when coupled to an IFS (Figure $2 b$ ). Ex-situ dynamic light scattering (DLS) reveals a unimer-tomicelle transition in the range of 7.0-7.5 for BCP-1 (Supporting Information Figure SI3). ${ }^{41}$ Upon simultaneous injection of the promoter/DD pair $(20 \mathrm{mM}$ TRIS $(\mathrm{pH}=8.8) /$ $50 \mathrm{mM} \delta$-GL), distinct changes in the $\mathrm{pH}$ evolution are observed in the presence of the BCP. First, the pristine IFS shows a higher $\mathrm{pH}$ jump than the one containing the BCP. This is due the presence of protonated DEAEMA- $\mathrm{H}^{+}$segments that consume $\mathrm{OH}^{-}$of the promoter to switch solvophobic and induce self-assembly. Even if acid/base reactions are among the simplest chemical reactions, it becomes evident that injection of insufficient promoter prevents the formation of self-assembled structures. Second, a buffer plateau appears around $\mathrm{pH} \approx 7.2$ during the $\mathrm{pH}$ decay, which corresponds to the $\mathrm{p} K_{\mathrm{a}}$ value of the DEAEMA and whose duration depends on the amount of DEAEMA. We further discuss the influence of the BCP concentration below. More importantly, the light scattering intensity $\left(I_{90^{\circ}}\right)$ displays a similar plateau-like profile, following the $\mathrm{pH}$ profile with a slight offset. This slight delay arises from kinetic retardation of the micelle-to-unimer-to-micelle transitions $\left(t_{\mathrm{d} 1}\right.$ and $t_{\mathrm{d} 2} ;$ Scheme $\left.1 \mathrm{~b}\right)$. Higher light scattering originates from larger structures and indicates the presence of self-assemblies, that are identified as spherical micelles by cryogenic transmission electron microscopy (cryo-TEM, Figure 2i). Simultaneous in-situ hydrodynamic size distribution analysis by DLS reveals unimers at the start $\left(\left\langle R_{\mathrm{h}}\right\rangle_{\mathrm{w}} \approx 3 \mathrm{~nm}\right)$, subsequent micelle formation and stable micelles $\left(\left\langle R_{\mathrm{h}}\right\rangle_{\mathrm{w}} \approx 12\right.$ $\mathrm{nm})$, and disassembly after $10 \mathrm{~min}$, coinciding with the drop in scattering intensity (Figure 2c). The distributions correspond to the ones measured ex-situ (Supporting Information Figure SI3). We exemplify temporal programming by changing the $\delta$ GL concentration, which allows regulating the self-assembly lifetimes $\left(t_{\mathrm{SA}-\mathrm{follow}}\right)$ in the range of minutes (Figure $2 \mathrm{~d}, \mathrm{e}$ ). During this process and in the plateau of stable self-assemblies, there is a constant exchange of $\mathrm{H}^{+} / \mathrm{OH}^{-}$between the various components, that is, the buffer molecules as promoter, the liberated acid and the DEAEMA units as interacting selfassembling system. At longer time scales the dynamic reprotonation by the liberated deactivating acid takes over, and deactivates the self-assembly by protonating the DEAEMA units, as well as protonates all TRIS- $\mathrm{NH}_{2}$ promoter molecules. Order-of-magnitude changes can be achieved conveniently using other DDs as shown below for BCP-2.

Next we change to BCP-2 containing a majority fraction of switchable DEAEMA (>90 wt \%), being in the so-called crewcut regime. ${ }^{42}$ BCP-2 provides the advantage that the typically larger sizes formed by crew-cut aggregates, in particular rod-like structures and vesicles, are macroscopically visible due to turbidity. DLS indeed shows larger sizes of $\left\langle R_{\mathrm{h}}\right\rangle_{\mathrm{w}} \approx 30 \mathrm{~nm}$ and cryo-TEM evidences vesicles (Figure 2i; Supporting Information SI3). This allows (i) following the temporal self-regulation by the naked eye (Figure 2f; Supporting Information Movie SI1) and (ii) simplifies the experiments as turbidity can be directly used to quantify the temporal evolution. Figure $2 \mathrm{f}$ displays snapshots of BCP-2 programmed with different $\delta$-GL concentrations to decay at times between 5 to $30 \mathrm{~min}$ 
a

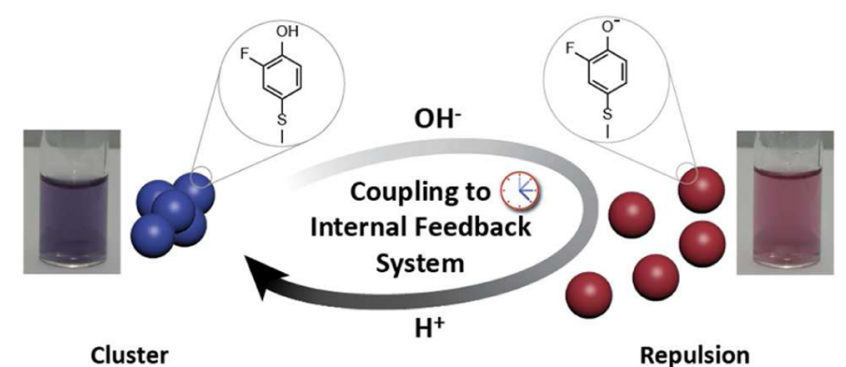

Cluster

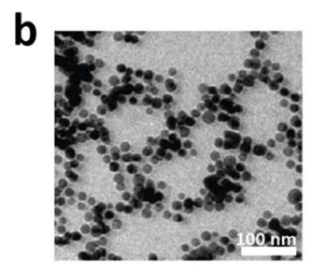

e

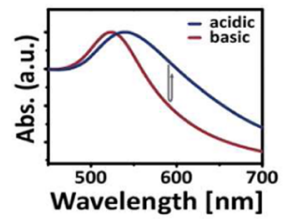

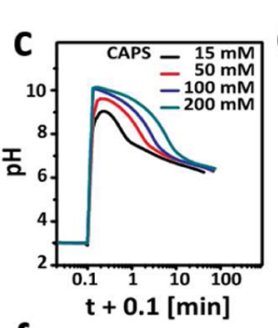

$f$
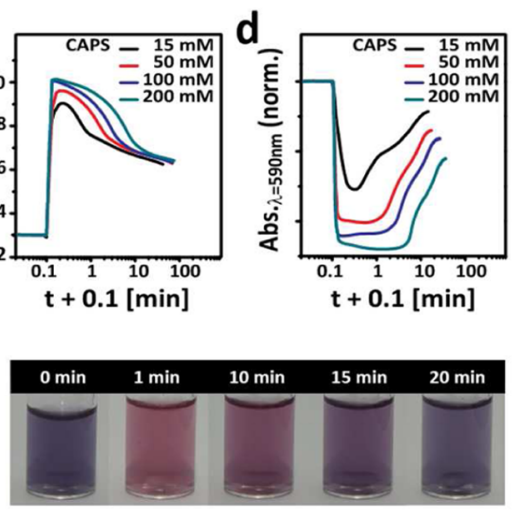

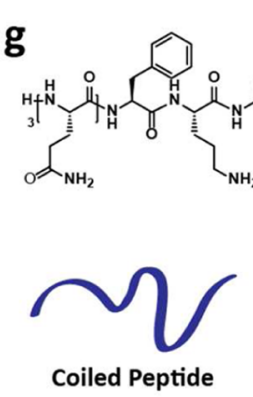

$h$

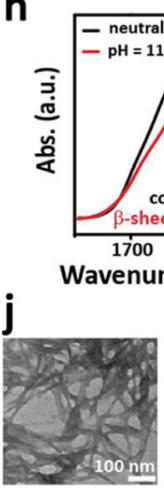

k
(N)

$\mathrm{OH}^{-}$

$\mathrm{OH}^{-}$

Coupling to Internal Feedback System

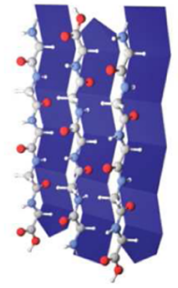

Antiparallel $\beta$-sheet Nanofibril

$\mathrm{H}^{+}$
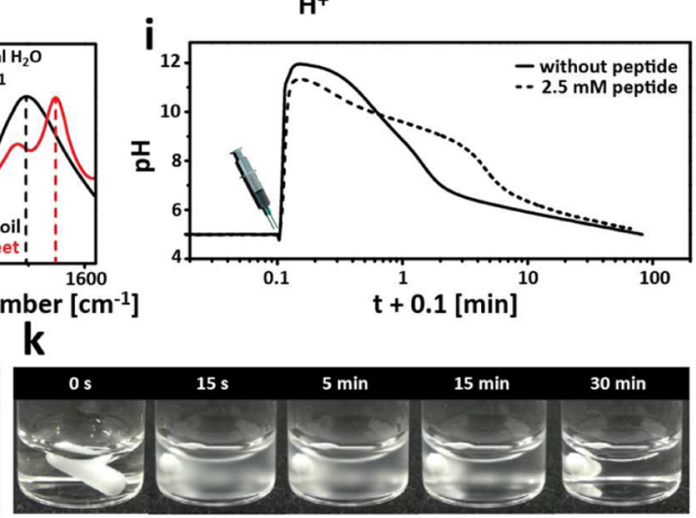

Figure 3. General ability for temporal programming of self-assembly lifetimes demonstrated for Au-NPs and oligopeptides. (a) Phenol-functionalized $\mathrm{Au}-\mathrm{NPs}$ show clusters at acidic $\mathrm{pH}$, which reversibly break due to electrostatic repulsion at high $\mathrm{pH}$ when subjected to suitable IFS. (b) Cryo-TEM illustrating Au-NP clusters at $\mathrm{pH}=5$. ( $\mathrm{c}-\mathrm{d}$ ) Temporal programming using different buffer strength of CAPS at constant concentration of $200 \mathrm{mM}$ MF as DD. $\mathrm{pH}$ profiles (c) and de/reclustering monitored via absorbance at $590 \mathrm{~nm}$ (d). (e) UV-vis spectra at acidic and alkaline pH showing the blue $(539 \mathrm{~nm})$ and red plasmon peak $(525 \mathrm{~nm})$. (f) Snapshot series at $60 \mathrm{mM}$ CAPS and $100 \mathrm{mM}$ MF (Supporting Information Movie SI2). (g) Structure and temporally programmed reversible switching of an oligopeptide from coil-to- $\beta$-sheet-to-coil traced by (h) FTIR spectroscopy, (i) pHprofile, (j) TEM of nanofibrils formed at alkaline $\mathrm{pH}$, and $(\mathrm{k})$ macroscopic turbidity as seen in the snapshot series (Supporting Information Movie SI3). Conditions: $2.5 \mathrm{mM}$ oligopeptide, $25 \mathrm{mM} \mathrm{NaOH}$, and $100 \mathrm{mM} \beta$-Bl.

(Supporting Information Movie SI1 and Figure SI4). Much longer lifetimes can be reached by changing the $\mathrm{DD}$ to $\beta$-BL ( $\beta$-butyrolactone) and $\varepsilon$-CL, allowing transient states of $3 \mathrm{~h}$ and 1 day (Figure $2 \mathrm{~g}$ ). This shift in time scale reflects the molecularly controlled decomposition kinetics of the pure compounds (Figure 1a) and demonstrates the unique ability to program self-regulation over four orders of magnitude in time.

Repeated jumps are possible upon reinjection of the IFS as illustrated for different concentrations of BCP-1 (Figure 2h). We perform this reactivation to exclude any negative effects of the promoter/deactivator pairs on the self-assembling components. The creation of stable oscillations is beyond the focus of this contribution. Interestingly, the $\mathrm{pH}$ jumps decrease and the curves show more pronounced plateaus (lifetimes of the selfassembly are longer) for higher BCP concentrations. The decrease in the $\mathrm{pH}$ jump is caused by the consumption of the promoter by higher concentrations of the protonated DEAEMA- $\mathrm{H}^{+}$segments, whereas the longer plateau is associated with two phenomena. First, the ester hydrolysis is base-catalyzed and occurs more quickly at higher $\mathrm{pH}$. Second, more DEAEMA units cause a longer buffering region around neutral $\mathrm{pH}\left(\mathrm{pK}_{\mathrm{a}} \approx 7.2\right)$ during the decay, stabilizing the hydrolysis of the DD at low speed before the acid-catalyzed decomposition takes over. The overall $\mathrm{pH}$ jump and plateau length decrease for repeated jumps due to remaining excess of DD.

Next, we demonstrate the general applicability to selfregulate and temporally program other self-assembling systems by applying IFSs to (i) gold nanoparticles (Au-NPs) and program optical plasmon effects by reversible deaggregation/ aggregation and (ii) oligopeptides undergoing reversible $\beta$ sheet nanofibrillization. Both material classes are cornerstones for advanced materials with applications in optical sensors, conducting nanowires and for biomedical applications. ${ }^{1,2,5,18}$

We use Au-NPs $\left(\langle R\rangle_{\mathrm{n}, \mathrm{TEM}}=3.9 \mathrm{~nm}\right)$ functionalized with a phenol-ligand, ${ }^{26}$ which undergoes deprotonation at high $\mathrm{pH}$ $\left(\mathrm{p} K_{\mathrm{a}} \approx 8.3\right)$, inducing declustering via electrostatic repulsion (Figure 3a). This can be followed macroscopically by a color change from blue to red due to the loss of interparticle coupling of the plasmons, as further quantified by UV-vis (Figure 3e, Supporting Information Movie SI2). Cryo-TEM depicts typical clusters at acidic conditions (Figure $3 b$ ). The weak acidity of the phenol group requires more alkaline buffers (CAPS (3(cyclohexylamino)-1-propanesulfonic acid), $\mathrm{pH}=11$ ) for sufficient ionization and to trigger the transition. In addition to changing the buffer, we now show temporal programming using a variation in buffer strength while maintaining the same concentration of DD (200 mM MF). The evolution is conveniently monitored using simultaneous $\mathrm{pH}$ measurement and optical absorption at $590 \mathrm{~nm}$ (largest difference between both spectra) to follow the dissolution of the Au-NP clusters (Figure $3 \mathrm{c}-\mathrm{f}$ ). The differences in the $\mathrm{pH}$ jump height reflect the increased concentration of switchable units and the in-situ absorption/pH measurements demonstrate successful programming on time scales from ca. 15 to $45 \mathrm{~min}$ upon increasing the buffer strength of the promoter from 15 to $200 \mathrm{mM}$. Differences in absorption at start and end are mainly due to dilution effects based on adding the IFS. Supporting 
Information Movie SI2 compares multiple injects of a fast DD ( $\delta$-GL) vs comparably slower MF, thereby showing the possibility of repeated activation and tuning of $t_{\mathrm{SA} \text {-follow }}$ using different DDs.

Even very delicate self-assemblies can be programmed in time as shown for an oligopeptide that undergoes a distinct isotropic-to-nematic transition due to base-induced fibrillization caused by switching its conformation from coil to $\beta$-sheets (Figure $3 \mathrm{~g}-\mathrm{k}$ ). ${ }^{43}$ The particular peptide displays a transition at $\mathrm{pH} \approx 7.6$, due to deprotonation of the amine groups and either forms nematic fluids $(>0.9 \mathrm{mM})$ or nematic gels $(>6.6 \mathrm{mM})$. We achieve temporal programming using $\mathrm{NaOH}$ as promoter and $\beta$-BL as DD. The formation of nanofibrils goes along with macroscopically observable turbidity (Figure 3k; Supporting Information Movie SI3). After $30 \mathrm{~min}$, the turbidity vanishes and a solution of original clarity is obtained, indicating the complete redissolution of the nanofibrils, again corresponding to the transient $\mathrm{pH}$ profile. TEM images and FTIR spectroscopy verify the intermediate presence of $\beta$-sheet-based peptide nanofibrils and confirm the programming of a transient selfassembled nanofibrillar state.

In summary, we presented a general concept to program the time domain of $\mathrm{pH}$-switchable self-assemblies over four orders of magnitude and equip them with a mechanism for autonomous self-regulation in closed systems. We realize this by controlling the kinetics of assembly and disassembly reaction, and more precisely by starving the availability of the deactivator using a dormant species. This conceptual approach enables a new level of control for switchable self-assembling systems and allows advancements toward more dynamic materials, spawning self-regulating and time-programmed (self-erasing) memory functions. We expect potential applications in photonic memories, self-erasing patterns, burst release hydrogels for delivery applications, and for noninvasive, in-situ analysis of self-assembly processes, for example, in a closed liquid-cell TEM, confocal and super-resolution microscopy and scattering experiments. The approach can be generalized by expanding to dormant deactivators releasing base and other dormant species (e.g., chelators, redox-active compounds). We reason that the balanced combination of different dormant activators and deactivators will allow to increase control over the system by also controlling the activation time scale $\left(v_{1}\right)$ and programming an "on" time. Even though not targeted by us, the increasing understanding of dormant species and strengthening artificial feedback mechanisms may lead to new designs for chemical oscillators. The successful orchestration of the time domain, and understanding self-regulation and artificial feedback mechanisms of diverse kinds affords the needed counterpart to widely pursued efforts toward ever more complex 3D hierarchical self-assemblies in space. The combination of both is of fundamental interest to promote and understand complex and more dynamic systems. For our systems, one of the most intriguing challenges will be to couple the time-scale of the self-assembly state to the induction of a secondary time-dependent process to at some point approach nonlinear evolutions.

Experimental Section. General Procedure for Temporal Programming of Self-Regulating Self-Assemblies. The calculated amounts of promoter and dormant deactivator solutions are loaded into gastight microsyringes and simultaneously injected into the vessel containing the self-assembling components. Liquid dormant deactivators (DDs) are injected directly, whereas solid DDs are dissolved close to their saturation concentration in DMSO at $0.5 \mathrm{~g} / \mathrm{mL}$. Depending on the self-assembling system, the temporal evolution is monitored via $\mathrm{pH}$ measurements, static and dynamic light scattering, as well as transmission and absorption measurements.

Further details can be found in the Supporting Information.

\section{ASSOCIATED CONTENT}

\section{Supporting Information}

Materials and experimental details, Figures SI1-4, Movies SI13 can be found in the Supporting Information. This material is available free of charge via the Internet at http://pubs.acs.org.

\section{AUTHOR INFORMATION}

\section{Corresponding Author}

*walther@dwi.rwth-aachen.de.

\section{Notes}

The authors declare no competing financial interest.

\section{ACKNOWLEDGMENTS}

We acknowledge funding by the DFG in grant agreement WA3084/4-1 and in the framework of the SFB 985 "Functional Microgels and Microgel Systems". A.W. acknowledges continuous support from M. Möller.

\section{REFERENCES}

(1) Aida, T.; Meijer, E. W.; Stupp, S. I. Science 2012, 335, 813-817.

(2) Grzelczak, M.; Vermant, J.; Furst, E. M.; Liz-Marzán, L. M. ACS Nano 2010, 4, 3591-3605.

(3) Mann, S. Nat. Mater. 2009, 8, 781-792.

(4) Whitesides, G. M.; Grzybowski, B. Science 2002, 295, 2418-2421.

(5) Glotzer, S. C.; Solomon, M. J. Nat. Mater. 2007, 6, 557-562.

(6) Gröschel, A. H.; Walther, A.; Löbling, T. I.; Schacher, F. H.; Schmalz, H.; Müller, A. H. E. Nature 2013, 503, 247-251.

(7) Liu, K.; Nie, Z.; Zhao, N.; Li, W.; Rubinstein, M.; Kumacheva, E. Science 2010, 329, 197-200.

(8) Rupar, P. A.; Chabanne, L.; Winnik, M. A.; Manners, I. Science 2012, 337, 559-562.

(9) Fukino, T.; Joo, H.; Hisada, Y.; Obana, M.; Yamagishi, H.; Hikima, T.; Takata, M.; Fujita, N.; Aida, T. Science 2014, 499-504.

(10) Cui, H.; Chen, Z.; Zhong, S.; Wooley, K. L.; Pochan, D. J. Science 2007, 317, 647-650.

(11) Bates, F. S.; Hillmyer, M. A.; Lodge, T. P.; Bates, C. M.; Delaney, K. T.; Fredrickson, G. H. Science 2012, 336, 434-440.

(12) Chen, Q.; Bae, S. C.; Granick, S. Nature 2011, 469, 381-384.

(13) Gröschel, A. H.; Schacher, F. H.; Schmalz, H.; Borisov, O. V.; Zhulina, E. B.; Walther, A.; Müller, A. H. E. Nat. Commun. 2012, 3, 710.

(14) Gilroy, J. B.; Gädt, T.; Whittell, G. R.; Chabanne, L.; Mitchels, J. M.; Richardson, R. M.; Winnik, M. A.; Manners, I. Nat. Chem. 2010, 2, $566-570$.

(15) Hermans, T. M.; Broeren, M. A. C.; Gomopoulos, N.; van der Schoot, P.; van Genderen, M. H. P.; Sommerdijk, N. A. J. M.; Fytas, G.; Meijer, E. W. Nat. Nano. 2009, 4, 721-726.

(16) Walther, A.; Drechsler, M.; Rosenfeldt, S.; Harnau, L.; Ballauff, M.; Abetz, V.; Müller, A. H. E. J. Am. Chem. Soc. 2009, 131, 47204728.

(17) Morris, K. L.; Chen, L.; Raeburn, J.; Sellick, O. R.; Cotanda, P.; Paul, A.; Griffiths, P. C.; King, S. M.; O’Reilly, R. K.; Serpell, L. C.; Adams, D. J. Nat. Commun. 2013, 4, 1480.

(18) Boekhoven, J.; Stupp, S. I. Adv. Mater. 2014, 26, 1642-1659.

(19) Kang, Y.; Walish, J. J.; Gorishnyy, T.; Thomas, E. L. Nat. Mater. 2007, 6, 957-960.

(20) Valkama, S.; Kosonen, H.; Ruokolainen, J.; Haatainen, T.; Torkkeli, M.; Serimaa, R.; ten Brinke, G.; Ikkala, O. Nat. Mater. 2004, 3, 872-876. 
(21) Park, S.; Lee, D. H.; Xu, J.; Kim, B.; Hong, S. W.; Jeong, U.; Xu, T.; Russell, T. P. Science 2009, 323, 1030-1033.

(22) Walther, A.; Bjurhager, I.; Malho, J.-M.; Pere, J.; Ruokolainen, J.; Berglund, L. A.; Ikkala, O. Nano Lett. 2010, 10, 2742-2748.

(23) Yoshida, R. Adv. Mater. 2010, 22, 3463-3483.

(24) Rabai, G.; Kustin, K.; Epstein, I. R. J. Am. Chem. Soc. 1989, 111, 3870-3874.

(25) Padirac, A.; Fujii, T.; Estévez-Torres, A.; Rondelez, Y. J. Am. Chem. Soc. 2013, 135, 14586-14592.

(26) Lagzi, I.; Kowalczyk, B.; Wang, D.; Grzybowski, B. A. Angew. Chem., Int. Ed. 2010, 49, 8616-8619.

(27) Rabai, G.; Orban, M.; Epstein, I. R. Acc. Chem. Res. 1990, 23, $258-263$.

(28) Poros, E.; Horváth, V.; Kurin-Csörgei, K.; Epstein, I. R.; Orbán, M. J. Am. Chem. Soc. 2011, 133, 7174-7179.

(29) Fialkowski, M.; Bishop, K. J. M.; Klajn, R.; Smoukov, S. K.; Campbell, C. J.; Grzybowski, B. A. J. Phys. Chem. B 2006, 110, 24822496.

(30) Grzybowski, B. A.; Stone, H. A.; Whitesides, G. M. Nature 2000, 405, 1033-1036.

(31) Timonen, J. V. I; Latikka, M.; Leibler, L.; Ras, R. H. A.; Ikkala, O. Science 2013, 341, 253-257.

(32) Boekhoven, J.; Brizard, A. M.; Kowlgi, K. N. K.; Koper, G. J. M.; Eelkema, R.; van Esch, J. H. Angew. Chem., Int. Ed 2010, 49, 48254828.

(33) Debnath, S.; Roy, S.; Ulijn, R. V. J. Am. Chem. Soc. 2013, 135, 16789-16792.

(34) "Dormant" as defined by Oxford Dictionaries. www. oxforddictionary.com (accessed Aug 10, 2014).

(35) Walther, A.; Yuan, J.; Abetz, V.; Müller, A. H. E. Nano Lett. 2009, 9, 2026-2030.

(36) Hao, J.; Hoffmann, H.; Horbaschek, K. J. Phys. Chem. B 2000, 104, 10144-10153.

(37) Adams, D. J.; Butler, M. F.; Frith, W. J.; Kirkland, M.; Mullen, L.; Sanderson, P. Soft Matter 2009, 5, 1856-1862.

(38) Cardoso, A. Z.; Alvarez Alvarez, A. E.; Cattoz, B. N.; Griffiths, P. C.; King, S. M.; Frith, W. J.; Adams, D. J. Faraday Discuss. 2013, 166, 101-116.

(39) Lagzi, I.; Wang, D.; Kowalczyk, B.; Grzybowski, B. A. Langmuir 2010, 26, 13770-13772.

(40) Kovacs, K.; Mcllwaine, R. E.; Scott, S. K.; Taylor, A. F. J. Phys. Chem. A 2007, 111, 549-551.

(41) Vamvakaki, M.; Papoutsakis, L.; Katsamanis, V.; Afchoudia, T.; Fragouli, P. G.; Iatrou, H.; Hadjichristidis, N.; Armes, S. P.; Sidorov, S.; Zhirov, D.; Zhirov, V.; Kostylev, M.; Bronstein, L. M.; Anastasiadis, S. H. Faraday Discuss. 2005, 128, 129-147.

(42) Walther, A.; Goldmann, A. S.; Yelamanchili, R. S.; Drechsler, M.; Schmalz, H.; Eisenberg, A.; Müller, A. H. E. Macromolecules 2008, 41, 3254-3260.

(43) Aggeli, A.; Bell, M.; Carrick, L. M.; Fishwick, C. W. G.; Harding, R.; Mawer, P. J.; Radford, S. E.; Strong, A. E.; Boden, N. J. Am. Chem. Soc. 2003, 125, 9619-9628. 